\title{
Ein achtsamer Blick auf den Achtsamkeits-Hype
}

\author{
Simon Schindler
}

Online publiziert: 23. Januar 2020

(C) Der/die Autor(en) 2020

Zusammenfassung Achtsamkeit liegt sowohl in der psychologischen Forschung als auch in vielen gesellschaftlichen Bereichen im Trend. Dieser Artikel wirft einen achtsamen Blick auf den aktuellen Forschungsstand. Dabei werden zum einen diverse methodische Limitationen bei der empirischen Untersuchung von Achtsamkeit deutlich, wodurch valide Aussagen über tatsächliche Effekte nach wie vor nur begrenzt möglich sind. Zum anderen wird eine Forschungslücke bei möglichen unerwünschten Auswirkungen identifiziert. So sind negative Konsequenzen beispielsweise auf interpersoneller und moralischer Ebene theoretisch plausibel herleitbar. Insgesamt kann ein Bedarf an weiterer Forschung konstatiert werden.

Schlüsselwörter Achtsamkeit · Meditation · Hype

\section{A mindful evaluation of the mindfulness hype}

\begin{abstract}
Mindfulness is currently a hot topic in psychological research as well as in many parts of the society. This article aims to critically evaluate the current state of research on this topic. First, several methodological limitations regarding the empirical investigation of mindfulness become apparent, restricting valid conclusions about the actual effects. In addition, a research gap on undesirable consequences is identified. Potential negative effects of mindfulness in interpersonal and moral domains, for example, are theoretically plausible and are discussed. In sum more high quality research is needed in many areas.
\end{abstract}

Keywords Mindfulness $\cdot$ Meditation · Hype

Dr. S. Schindler $(\bowtie)$

Institut für Psychologie, Univ. Kassel, Holländische Str. 36-38, 34127 Kassel, Deutschland

E-Mail: schindler@uni-kassel.de 


\section{Einleitung}

Schließen Sie Ihre Augen für eine Minute und werden Sie sich Ihres Atems bewusst. Beobachten Sie Ihre Empfindungen bei jedem Ein- und Ausatmen. Ihre Gedanken werden vermutlich schnell von Ihrer Atmung abschweifen. Nehmen Sie das einfach offen und mit Neugier wahr und akzeptieren Sie, wo auch immer Ihre Gedanken gerade sind. Erkennen Sie Ihre momentanen Gedanken als bloße, flüchtige Gedanken und Ihre Gefühle als bloße, flüchtige Gefühle - frei von jedem Urteil, voller Akzeptanz. Dann können Sie Ihre Aufmerksamkeit sachte auf Ihren Anker zurücklenken, auf die Erfahrung eines jeden Atemzuges, wie er ein- und ausströmt.

So kann eine typische Anleitung klingen, um sich in Achtsamkeit zu üben. Ein Blick in einen der vielen Achtsamkeits-Ratgeber verspricht vielfältige positive Folgen einer solchen Übung: Achtsamkeit bringt ,,mehr Ruhe und Entspannung, mehr Energie und Lebensfreude, ein höheres Maß an Selbstvertrauen und -akzeptanz, geringere Anfälligkeit gegenüber Stress, Depression, Angst, chronischen Schmerzen, Suchtverhalten und Immunschwäche“, und nicht zuletzt stärkt Achtsamkeit das „Mitgefühl mit sich selbst und anderen Menschen, aber auch mit unserem Planeten“ (Collard 2016, S. 7). Viele dieser positiven Auswirkungen können tatsächlich durch empirische Studien gestützt werden. Gemessen an der enorm gestiegenen Zahl der wissenschaftlichen Publikationen (mittlerweile weit über 1000; Van Dam et al. 2018), hat sich Achtsamkeit in den letzten zwanzig Jahren in der psychologischen Forschung zu einem hot topic entwickelt (Brown et al. 2015). Es scheint kaum ein Problem zu geben, zu dessen Lösung Achtsamkeit nicht beitragen kann wissenschaftlich fundiert.

Auch in der breiten Öffentlichkeit ist Achtsamkeit schwer angesagt: Sämtliche Zeitungen haben bereits darüber berichtet. Ganze Zeitschriften (wie Flow oder Happinez) widmen sich diesem Thema. Sucht man mit Google nach Videos zu „Achtsamkeit“, werden über 150.000 Treffer angezeigt - zu ,mindfulness“ sind es über 16Mio. Eine Suche über Thalia.de zu „Achtsamkeit“ in der Rubrik „Ratgeber“ ergab knapp 700 Treffer - davon erschienen allein in den letzten drei Jahren jährlich mehr als 150 Bücher. Eine Achtsamkeits-App (Calm) war die Apple App des Jahres 2017. In Psychotherapien wird Achtsamkeit angewandt, Krankenkassen empfehlen mehr Achtsamkeit, an Schulen und im Militär wird Achtsamkeit kultiviert, und große Unternehmen (wie SAP, Nike oder Google) bieten Achtsamkeitstrainings für ihre Beschäftigten an. Diesem Trend entsprechend wird Achtsamkeit auch für den Coaching-Bereich beworben, sowohl für den Coach als auch für den Klienten (Bosch und Michel 2016).

Entgegen dem menschlichen Verlangen nach eindeutiger, unumstößlicher Wahrheit, ist wissenschaftliche Erkenntnis ihrem Wesen nach nie endgültig, sondern immer nur vorläufig (Popper 1963). Gerade der scheinbar makellose Ruf von Achtsamkeit und die hohe Popularität nähren aus Forschungsperspektive daher den Verdacht der Übertreibung. Ein nüchterner, prüfender, ja achtsamer Blick auf den aktuellen Forschungsstand ist gefordert, um eventuelle Fehl- bzw. Überinterpretationen und Limitationen zu identifizieren. So sind in der letzten Zeit vermehrt wissenschaftli- 
che Artikel erschienen, die sich kritisch mit Achtsamkeit und der Forschung dazu auseinandersetzen (z. B. Van Dam et al. 2018). Der vorliegende Artikel soll dazu beitragen, den Achtsamkeits-Hype kritisch zu beleuchten, und widmet sich zunächst der empirischen Ebene; hier werden Herausforderungen und Limitationen bei der empirischen Untersuchung von Achtsamkeit abgehandelt. Im Anschluss geht es um die theoretische Ebene; hier werden mögliche Ideen und erste Evidenz präsentiert, die auch unerwünschte Effekte von Achtsamkeit, beispielsweise im Bereich von Motivation und Moral, zeigen. Insgesamt soll es in diesem Artikel nicht darum gehen, die Achtsamkeitspraxis als solche zu diskreditieren. Ziel ist es vielmehr, den Erkenntnisgewinn durch zukünftige Forschung zu fördern.

\section{Herausforderungen und Limitationen bei der empirischen Untersuchung}

Ohne Zweifel ist die schiere Zahl der Forschungsarbeiten zu Achtsamkeit beeindruckend hoch. Dennoch ist Achtsamkeit, entgegen dem Ruf, in vielen Bereichen unterforscht, und die Rufe nach qualitativ hochwertigeren Studien werden lauter (z. B. Creswell 2017; Rosenkranz et al. 2019). Der Erkenntnisgewinn durch einzelne Studien ist immer durch die Limitationen der angewandten Methoden bedingt. Das gilt grundsätzlich für alle empirische Forschung. Es ist hierbei wichtig, korrekt einzuschätzen, wie gravierend diese Limitationen in einem Forschungsbereich sind. Es sei an dieser Stelle angemerkt, dass viele der folgenden Punkte für quantitative Forschung im Allgemeinen zutreffen und nicht auf die Achtsamkeitsforschung beschränkt sind.

\subsection{Vieldeutigkeit und Operationalisierung}

Im Alltag lassen wir häufig unsere Gedanken unbewusst schweifen, folgen dem Autopiloten und schieben unangenehme Gedanken und Gefühle beiseite. Achtsamkeit steht im Kontrast dazu. Hier geht es um eine Erfahrung des Hier-und-Jetzt, um ein Bewusstsein für den Moment. Aber was bedeutet das ursprünglich aus dem Buddhismus kommende, in der Forschung weitgehend säkularisierte Konzept genau? Obwohl Diskussionen um Definitionen nur indirekt zum wissenschaftlichen Erkenntnisgewinn beitragen, sind Definitionen entscheidend für eine konstruktive und produktive Kommunikation innerhalb der Forschung, auch mit der Öffentlichkeit. Es bietet sich deshalb an, einheitliche Definitionen zu verwenden. Wie bei anderen psychologischen Konstrukten (z. B. Intelligenz oder Empathie) gibt es nicht nur im öffentlichen, sondern auch im wissenschaftlichen Diskurs verschiedene Definitionen von Achtsamkeit. Eine der gängigsten beschreibt Achtsamkeit als eine bestimmte Form der Aufmerksamkeit, die absichtsvoll ist, sich auf den gegenwärtigen Moment bezieht und dabei nicht wertend ist (Kabat-Zinn 1990). Eine andere Definition begreift Achtsamkeit als ein Zwei-Komponenten-Modell der Selbstregulation von Aufmerksamkeit und der Orientierung an momentaner Erfahrung (Bishop et al. 2004). Die existierenden Definitionen implizieren meist mehrere, teilweise un- 
terschiedliche mentale Facetten, die meist selbst wiederum komplexe Konstrukte darstellen (Nilsson und Kazemi 2016).

Für weitere Unklarheit sorgt der Umstand, dass die Begriffe Achtsamkeit und Meditation häufig in einen Topf geworfen werden (z. B. Condon et al. 2013). Dabei gibt es viele verschiedene Arten der Meditation, die sich in ihrer Herangehensweise und in den theoretischen Grundannahmen teilweise stark unterscheiden. So soll beispielsweise durch loving-kindness Meditation (Salzberg 1995) Mitgefühl und Vergebung kultiviert werden, was bei typischen Achtsamkeitsübungen nicht explizit als Ziel formuliert wird. Ein Problem, das mit der Vieldeutigkeit von Achtsamkeit zusammenhängt, bezieht sich auf das ,erfolgreiche“ Erreichen von Achtsamkeit. Wann genau kann man sicher sein, einen achtsamen Zustand erreicht zu haben? Und gelingt ein achtsamer Zustand schon bei der ersten kurzen Übung oder braucht es mehrere hundert Stunden Erfahrung? Je nach Forschungsbereich und Autor/innen und je nach zugrunde gelegter Definition werden hier unterschiedliche Annahmen getroffen (vgl. Grossmann 2019). Das birgt mitunter die Gefahr, dass ausbleibende oder nicht wünschenswerte Effekte einfach damit erklärt werden können, dass es sich ja nicht um ,richtige“ Achtsamkeit gehandelt hat oder lediglich nicht genug geübt wurde. Aus wissenschaftlicher Sicht ist es allerdings fruchtlos, ein Konzept über seine Auswirkungen zu definieren.

Eine weitere Schwierigkeit, die sich unter anderem aus dem fehlenden Konsens einer einheitlichen Definition ergibt, betrifft die Frage der Messbarkeit oder Operationalisierung. Der Großteil der psychologischen Forschung zu Achtsamkeit beruht auf der Verwendung von Selbstbericht-Fragebögen (Van Dam et al. 2018), einer in der Psychologie gängigen Methode, um Zusammenhänge zwischen verschiedenen Konstrukten zu untersuchen. Derzeit gibt es nicht weniger als elf verschiedene Selbstbericht-Fragebögen (Bergomi et al. 2013). Manche dieser Fragebögen erfassen Achtsamkeit als eindimensionales Konstrukt (Brown und Ryan 2003), andere beinhalten fünf Subdimensionen (Baer et al. 2006). Manche erfassen Achtsamkeit als momentanen Zustand, andere als Persönlichkeitseigenschaft. Manche Fragebögen zielen direkt auf die Erfassung von Achtsamkeit ab, andere erfassen die Abwesenheit von Achtsamkeit und schließen darüber auf ihre (fehlende) Ausprägung. Zusätzlich gibt es Skalen, die Achtsamkeit als eine von mehreren Subdimensionen eines übergreifenden Konstrukts erfassen (z. B. self-compassion; für Kritik siehe Pfattheicher et al. 2017). Jenseits dieser mangelnden Konstruktvalidität setzen akkurate Messungen über Selbstbericht-Fragebögen eine akkurate Selbstbeobachtung voraus. Motivationale Verzerrungen beim Antwortverhalten (wie soziale Erwünschtheit) sowie ein fehleranfälliges Gedächtnis machen das zum Problem. Dazu kommt, dass Items in den Fragebögen oftmals mehrdeutig formuliert sind und nicht klar ist, welche mentalen Facetten und Prozesse genau gemeint sind (vgl. Grossman 2019).

\subsection{Kausalität}

Die größte Limitation von reinen Fragebogenstudien (und auch den meisten Arbeiten aus dem neurowissenschaftlichen Bereich; z. B. Tang et al. 2015) zu Achtsamkeit ist jedoch deren korrelative Datenbasis. Schlussfolgerungen auf Ursache und Wirkung bzw. Wirkrichtung sind damit häufig spekulativ. In den letzten zwanzig Jah- 
ren gab es daher (vor allem im klinischen Bereich) verstärkt die Anstrengung, die Auswirkungen verschiedener Achtsamkeitsinterventionen zu untersuchen. Die wohl bekannteste und am häufigsten untersuchte Intervention ist das Mindfulness-Based Stress Reduction (MBSR) Programm von Jon Kabat-Zinn (1982). Das Programm richtet sich in erster Linie an Patient/innen mit verschiedenen Leiden, wie z. B. chronischen Schmerzen, Depressionen, Essstörungen oder Drogenabhängigkeit, dauert üblicherweise acht Wochen und umfasst verschiedene Übungen, wie Atemübungen, Body-Scans und Hatha Yoga. Andere Achtsamkeitsinterventionen dauern dagegen nur zwei bis drei Wochen (Lim et al. 2015), drei bis vier Tage (Creswell et al. 2014) oder auch nur zehn bis zwanzig Minuten (Papies et al. 2015). Diese Übungen unterscheiden sich zwar stark in ihrer Dauer und ihrem inhaltlichen Fokus, sie fallen in der Literatur aber alle unter den Sammelbegriff Achtsamkeit. Entsprechend der Vieldeutigkeit des Achtsamkeitsbegriffs gibt es also auch auf Interventionsebene eine Operationalisierungsvielfalt. Das erschwert die Vergleichbarkeit der Studien nicht nur hinsichtlich ihrer Wirkungen, sondern auch der angenommenen Prozesse.

Um die tatsächlichen Auswirkungen von Achtsamkeit und die zugrundeliegenden Prozesse angemessen zu untersuchen, bedarf es zudem experimenteller Designs mit randomisierter Zuweisung der Versuchspersonen zur Achtsamkeits- und einer adäquaten Kontrollbedingung. Dies ist bei dem Großteil der (vor allem klinischen) Studien zu Achtsamkeitsinterventionen nicht gegeben (Dimidjian und Segal 2015). Dabei ist die Frage der Kontrollgruppe eine entscheidende, denn es ist nur wenig überraschend, wenn eine Achtsamkeitsintervention im Vergleich zu keiner Behandlung (z.B. einer sog. wait list-Kontrollgruppe) die Entspannung erhöht und den Stresslevel senkt. Wichtiger wäre zu wissen, im Vergleich zu welchen anderen Interventionen Achtsamkeit besonders wirksam ist - oder eben auch nicht (z. B. Rosenkranz et al. 2019); wirkt eine Achtsamkeitsintervention beispielsweise besser als eine andere Entspannungs-Intervention, als Sport, als eine Massage oder als Sex?

In diesem Zusammenhang spielt der Placebo-Effekt eine wichtige Rolle. Bezogen auf Achtsamkeitsinterventionen bedeutet das, dass ein Effekt bei einer Person nicht durch die Intervention selbst auftritt, sondern durch den Glauben der Person an die Wirkung der Intervention. Homöopathen würden dazu vermutlich sagen: „Wer heilt, hat Recht". Ein solches Argument ist aus wissenschaftlicher Sicht Humbug. Denn es ist ja eben nicht klar, wer und was hier eigentlich genau geheilt hat (vorausgesetzt eine Heilung fand überhaupt statt), und eine solche Sichtweise kann vor allem im klinischen Bereich fatale Folgen für die Patient/innen haben (Johnson et al. 2018). Um die Auswirkungen von Achtsamkeitsinterventionen und deren Prozesse wirklich zu verstehen, bedarf es daher Placebo-Kontrollgruppen. Diese sind in der Achtsamkeitsforschung allerdings bislang die Ausnahme (z. B. Zeidan et al. 2015).

Häufig wird argumentiert, dass Achtsamkeitsübungen eine gewisse Erfahrung voraussetzen, um die gewünschten Effekte zu produzieren (z. B. Walsh und Shapiro 2006). Der Aspekt der Erfahrung ist für die Untersuchung der Wirksamkeit zentral, da sonst bei ausbleibendem oder gar gegenläufigem Effekt argumentiert werden kann, dass lediglich noch nicht ausreichend geübt wurde. Wann jedoch das Kriterium für ,,ausreichend“ erfüllt wäre, wird in der Regel nicht festgelegt. Mit anderen Worten: Es braucht ein klares Kriterium für die Falsifikation der Wirksamkeit. Um die Rolle von Erfahrung experimentell zu testen, müssen darüber hinaus mögliche 
Störungen durch Konfundierungen bedacht werden. Denn es ist anzunehmen, dass erfahrene Menschen bestimmte Merkmale wie beispielsweise ein von buddhistischen Werten geprägtes Weltbild teilen, in dem das Vermeiden von Leiden gegenüber allen Lebewesen eine zentrale Rolle spielt. Wenn nun eine Studie zu dem Ergebnis gelangt, dass Achtsamkeitsübungen bei der erfahrenen Gruppe im Vergleich zu einer ungeübten Gruppe zu mehr Mitgefühl und erhöhtem Hilfeverhalten führt, dann bleibt unklar, ob der Effekt tatsächlich auf die Übungen zurückzuführen ist oder auf das prosoziale Weltbild. Solche möglichen Konfundierungen erschweren es, die Rolle von Erfahrung herauszuarbeiten. Langzeit-Interventionsstudien mit randomisierter Zuweisung und angemessenen Kontrollgruppen sind hier ein Lösungsweg, der aufwendig ist und in diesem Forschungsbereich bislang nicht ausreichend realisiert wurde (Rosenkranz et al. 2019).

\subsection{Meta-Analysen}

$\mathrm{Zu}$ Achtsamkeitsinterventionen gibt es mittlerweile eine beeindruckend hohe Zahl an Meta-Analysen. Eine kurze Literaturrecherche ergab alleine für 2018 mehr als zwanzig Publikationen, die meta-analytisch vorgingen. Das Ergebnis einer MetaAnalyse gilt zurecht als wesentlich aussagekräftiger bzw. belastbarer als die Ergebnisse von Einzelstudien. Unter anderem reduziert sie die Gefahr der Rosinenpickerei, um bestimmte Aussagen zu belegen. Allerdings kann auch die Qualität von MetaAnalyse erheblich variieren.

In großen Teilen der Wissenschaftswelt gilt das Signifikanzniveau noch immer als ein hartes Kriterium, um die Verlässlichkeit wissenschaftlicher Befunde zu beurteilen. Die Jagd nach signifikanten Ergebnissen führt aber leider immer wieder dazu, dass Untersuchungen mit nicht-signifikanten Ergebnissen gar nicht erst das Licht der Öffentlichkeit erblicken und in der Schublade landen (Rosenthal 1979). Für die Aussagekraft von Meta-Analysen hat das gravierende Folgen: Da die Existenz vieler nicht-signifikanter Studien nicht bekannt ist, gehen diese nicht in die Meta-Analyse mit ein - damit gibt es eine Verzerrung zugunsten publizierter und damit signifikanter Ergebnisse (sog. publication bias). Ein Hinweis hierauf liegt vor, wenn die Zahl der publizierten Studien mit signifikanten Ergebnissen weitaus größer ist, als es die durchschnittliche Teststärke der Studien vermuten ließe. Hinweise auf einen solchen publication bias wurden beispielweise für den positiven Einfluss von Achtsamkeitsinterventionen auf die mentale Gesundheit gefunden, was eine Überschätzung der positiven Auswirkungen nahelegt (Coronado-Montoya et al. 2016). Bislang übliche statistische Verfahren, um solche Verzerrung zu erkennen und zu korrigieren, haben sich teilweise selbst als fehleranfällig erwiesen, weshalb neuere Methoden zu empfehlen sind (vgl. Carter et al. 2019). Um bestehende Probleme wie einen publication bias (oder auch p-hacking; Simmons et al. 2011) strukturell anzugehen, ist es bei vielen psychologischen Zeitschriften mittlerweile Standard, dass die Vorhersagen, die angestrebte Stichprobengröße sowie die Datenanalyse vor der Durchführung einer Studie präregistriert werden. Diese Maßnahmen sind generell für die Achtsamkeitsforschung zu empfehlen, wurden bisher aber nur unzureichend umgesetzt. 
Darüber hinaus ist die Vergleichbarkeit von Interventionsstudien aufgrund unterschiedlichster Operationalisierungen von Achtsamkeit oft eingeschränkt. Es erscheint dann wenig sinnvoll, diese Studien meta-analytisch zusammenzufassen. Hinzu kommt das erwähnte Problem mangelhafter Kontrollgruppen. Sehr wahrscheinlich ist der Achtsamkeitseffekt in Studien größer, die eine Kontrollgruppe ohne Intervention verwenden, als eine aktive Kontrollgruppe, in der die Versuchspersonen beispielsweise eine Entspannungsübung durchführen sollen (z. B. Jain et al. 2007). Insgesamt kann eine Meta-Analyse qualitativ nur so hochwertig sein, wie es die eingegangenen Studien selbst sind. Wenn also der Qualitätsstandard in einem bestimmten Forschungsfeld zu wünschen übriglässt, wird auch eine Meta-Analyse diesen Umstand nicht korrigieren können. Die aus Meta-Analysen resultierenden Effektstärken zu Achtsamkeitsinterventionen sind deshalb mit Vorsicht zu genießen.

\subsection{Motivationale Anreize}

Viele motivationale Anreize für Forschung stehen dem tatsächlichen Erkenntnisgewinn im Weg. Neben dem Anreiz, möglichst viel zu publizieren und signifikante Ergebnisse zu produzieren, sind Achtsamkeitsforscher/innen mit einem weiteren Interessenskonflikt konfrontiert, nämlich dann, wenn finanzielle Interessen hinter Achtsamkeitsinterventionen stehen. Erst kürzlich wurde eine viel zitierte Meta-Analyse zu Achtsamkeitsinterventionen im Gesundheitsbereich zurückgezogen (Gotink et al. 2015). Die betroffene Zeitschrift (PLoS ONE) begründete diesen Schritt unter anderem damit, dass der Editor befangen war, dass methodisch fragwürdig vorgegangen sei und dass zwei der Autor/innen an einem Institut angestellt sind bzw. waren, das mit Achtsamkeits-bezogenen Behandlungen Geld verdient - ein klarer Interessenskonflikt, den die Autoren zuvor verschwiegen hatten. Dieser Fall ist bislang eine Ausnahme und soll nicht den Eindruck einer gängigen Praxis erwecken. Allerdings lässt sich angesichts des enormen öffentlichen Interesses am Thema Achtsamkeit derzeit vermutlich viel Geld mit wissenschaftlich fundierten Achtsamkeitstrainings machen (Purser 2019) - das Interesse an tatsächlicher Erkenntnis durch Ergebnisoffenes Forschen wird dadurch höchst wahrscheinlich nicht steigen.

Ein weiterer motivationaler Anreiz bezieht sich auf das grundlegende menschliche Bedürfnis nach einem klaren, gültigen Weltbild, das insbesondere in schwierigen Zeiten Schutz bietet (Pyszczynski et al. 2015). Es ist plausibel, anzunehmen, dass der Glaube an die eigene Forschung und die Identifikation damit eine solche Funktion erfüllen kann (obwohl die empirische Überprüfung dieser Idee noch aussteht). In diesem Fall wird Achtsamkeit zu einer Art Religion, die Hoffnung auf die Lösung existentieller Probleme gibt. Forschung, die aus einem solchen Glauben resultiert, wird und soll im Wesentlichen nur das bestätigen, was für den Forschenden selbst längst schon als sicheres Wissen gilt. Folglich werden widersprüchliche Befunde ignoriert oder abgewertet. Was auf der einen Seite als allzu menschlich angesehen werden kann, hält auf der anderen Seite den wissenschaftlichen Erkenntnisgewinn in Grenzen. 


\section{Unerwünschte Effekte von Achtsamkeit}

Nachdem die Limitationen bei der empirischen Untersuchung von Achtsamkeit abgehandelt wurden, geht es nun um theoretische Ideen und empirische Evidenz, die unerwünschte Effekte von Achtsamkeit adressieren. Diese konzentrieren sich in der Literatur üblicherweise auf negative Erfahrungen während des Praktizierens, wie Unruhe, Angst, Unbehagen oder kognitive Erschöpfung (Creswell 2017). Insbesondere bei klinischen Patienten mit Angststörungen stellt sich damit die Frage, ob eine Achtsamkeits-basierte Therapie zur Behandlung der psychischen Krankheit eingesetzt werden sollte (Strauss et al. 2014).

Jenseits solcher negativen Erfahrungen beim Praktizieren selbst findet man lediglich eine Handvoll Artikel, die den makellosen Ruf von Achtsamkeit in Frage stellen. Eine erste Publikation dazu konnte in drei Experimenten zeigen, dass Versuchspersonen nach einer 15-minütigen Achtsamkeitsübung schlechter darin waren, gelernte Wörter korrekt zu identifizieren (Wilson et al. 2015). Die Autoren erklären den Befund mit der urteilsfreien und akzeptierenden Haltung bei Achtsamkeit. Hierdurch falle es schwerer, korrekt zwischen internalen und externalen Informationsquellen zu unterscheiden. Eine solche (Neben-) Wirkung dürfte die Anwendung von Achtsamkeitsübungen beispielsweise im Schulalltag eher unattraktiv machen.

Dass wenige solcher Publikationen zu finden sind, könnte natürlich daran liegen, dass Achtsamkeit tatsächlich kaum unerwünschte Effekte hat. Eine andere Möglichkeit ist, dass unerwünschte Effekte von Achtsamkeitsforscher/innen theoretisch von vorneherein ausgeschlossen und damit erst gar nicht untersucht wurden (siehe motivationale Anreize). Eine solche Perspektive impliziert ausnahmslos Win-WinSituationen. Das heißt, alles was z. B. für den Arbeitgeber gut ist, ist zwangsläufig für den Angestellten gut. Oder was für das Individuum gut ist, ist zwangsläufig für die Gesellschaft als Ganzes gut. Entgegen dieser vielleicht wünschenswerten Vorstellung lassen sich aus den angenommenen Wirkmechanismen von Achtsamkeit in verschiedenen Bereichen unerwünschte Auswirkungen ableiten.

\subsection{Motivation im Arbeitskontext}

Wie eingangs erwähnt, findet Achtsamkeit auch im Arbeits- und Organisationskontext starken Anklang: Immerhin zeigen Studien mit Angestellten, dass Achtsamkeitsinterventionen z. B. die Arbeitszufriedenheit erhöhen und Erschöpfungserscheinungen verringern (Hülsheger et al. 2013), die Schlafqualität verbessern (Hülsheger et al. 2015) und Vergeltungsimpulse gegenüber anderen Kolleg/innen abschwächen können (Long und Christian 2015). Das hat mutmaßlich mit der urteilsfreien und akzeptierenden Haltung zu tun, die durch Achtsamkeit kultiviert wird. Eine solche Haltung steht allerdings im Kontrast zur Leistungsmotivation, die eben dadurch entsteht, dass ein Zustand erreicht werden soll, der besser ist als der jetzige.

Eine Haltung völliger Akzeptanz dessen, was ist, impliziert aber auch, dass es keinen Grund gibt, etwas an der aktuellen Situation zu ändern. Diese Überlegungen führten Hafenbrack und Vohs (2018) zu der Hypothese, dass Achtsamkeitsübungen die Leistungsmotivation reduziert. Über sechs Studien hinweg fanden sie Evidenz für diese Idee: Die Motivation, an einer vorgegebenen Aufgabe zu arbeiten, war 
niedriger, wenn zuvor eine etwa 15-minütige Achtsamkeitsübung absolviert wurde. Versuchspersonen in den Kontrollgruppen sollten entweder ihre Gedanken frei schweifen lassen, Nachrichten lesen oder über vergangene Aktivitäten schreiben. Darüber hinaus fanden die Autoren weder einen positiven noch einen negativen Effekt auf die tatsächlich erbrachte Leistung. Hier ist unter anderem zu kritisieren, dass der Großteil der Studien online erhoben worden ist und damit nicht kontrolliert werden kann, was die Versuchspersonen während der Übung tatsächlich taten. Nichtsdestoweniger legen diese Ergebnisse nahe, dass bei Achtsamkeitsangeboten am Arbeitsplatz eine Reduktion der Leistungsmotivation bedacht werden sollte. Laut Hafenbrack und Vohs (2018) ist es ratsam, zu überlegen, zu welchem Zeitpunkt im Tageslauf die Achtsamkeitsübungen angeboten werden. In jedem Fall bekräftigt ihre Arbeit, dass Achtsamkeit und ihre Auswirkungen differenzierter betrachtet werden müssen (siehe auch Rupprecht et al. 2019).

\subsection{Prosoziale und moralische Reaktionen}

Neben dem Gesundheitsbereich wird Achtsamkeit insbesondere im zwischenmenschlichen Kontext weithin als nützlich verstanden. Erst kürzlich erschien eine Meta-Analyse, die über 13 Studien hinweg einen positiven Effekt von Achtsamkeitsinterventionen auf prosoziale Reaktionen zeigt (Donald et al. 2019). Die geringe Zahl an eingeflossenen Studien zeigt allerdings, dass dieser Forschungsbereich noch in den Kinderschuhen steckt (Creswell 2017). Die Autoren der Meta-Analyse räumen zudem ein, dass es Hinweise auf einen publication bias gibt, dass wegen methodischer Argumente auf p-hacking Analysen verzichtet wurde und dass die Achtsamkeitsinterventionen sehr unterschiedlich waren, was insgesamt die Aussagekraft einschränkt. Eine weitere Meta-Analyse über verschiedene Meditationspraktiken hinweg fand hingegen nur wenig Belege für einen prosozialen Effekt (Kreplin et al. 2018).

Anstatt darüber zu diskutieren, $o b$ es nun einen prosozialen Effekt von Achtsamkeit gibt oder nicht, sollte zunächst die theoretische Ebene im Fokus stehen: Warum sollte erhöhtes prosoziales Verhalten nach einer Achtsamkeitsübung überhaupt auftreten? Donald et al. (2019) führen mehrere mögliche Erklärungen an: Davon bezieht sich eine auf generell erhöhte Aufmerksamkeit und eine andere auf erhöhtes Mitgefühl. Beide Erklärungen führen je nach Situation zu verschiedenen Vorhersagen. Wenn Achtsamkeitsübungen generell die Aufmerksamkeit erhöhen, sollte erhöhtes prosoziales Verhalten unter Achtsamkeit nur dann zu beobachten sein, wenn dafür Aufmerksamkeit benötigt wird (z. B. in einer vollen Fußgängerzone oder unklarem Hilfebedürfnis). In Situationen, in denen keine erhöhte Aufmerksamkeit vonnöten ist, wäre gemäß dieser Erklärung kein Effekt zu erwarten. Wenn Achtsamkeit das Mitgefühl steigert, sollte einem Opfer z. B. von Gewalt verstärkt geholfen, aber gemäß neueren Befunden der Täter auch stärker bestraft werden (Pfattheicher et al. 2019). Die theoretisch weiterführende Frage ist hier also nicht, ob Achtsamkeit prosoziales Verhalten fördert, sondern was die Randbedingungen dafür sind. Solange die Erklärungen und theoretischen Annahmen für eine Vorhersage unklar sind, bleiben die Studien wenig Erkenntnis-bringend. 
Löst man sich von der Idee, dass Achtsamkeit die Lösung sämtlicher Probleme ist, so entstehen interessante und gesellschaftlich relevante Fragestellungen. So untersuchen Schindler et al. (2019) die Auswirkungen von Achtsamkeit auf moralische Reaktionen. Wir stützen uns dabei auf die empirisch relativ solide gestützte Annahme, dass Achtsamkeitsübungen die Fähigkeit verbessern, Emotionen zu regulieren, wodurch der handlungsleitende Einfluss von Emotionen geschwächt wird (vgl. Friese und Hofmann 2016). Im Kern ist das auf die urteilsfreie und akzeptierende Haltung zurückzuführen, die bei Achtsamkeitsübungen kultiviert wird. Wenn Achtsamkeit nun den Einfluss von Emotionen auf das Handeln schwächt, dann hat das im Falle von Emotionen wie Wut oder Rachegelüsten u. U. positive prosoziale Auswirkungen. Auf der anderen Seite führt dieser Mechanismus dazu, dass moralische Reaktionen geschwächt werden können, nämlich dann, wenn es um moralische Emotionen wie Schuld geht. Wenn andere durch unser Tun einen Schaden erleiden, entstehen Schuldgefühle, die die Motivation erzeugen, den Schaden wieder zu reparieren (z.B. Graton und Ric 2017). Ohne Schuldgefühle fehlt die Motivation zur Reparatur. Die Schlussfolgerung ist demnach: Achtsamkeitsübungen schwächen Schuld-basierte moralische Reaktionen.

In zwei Studien fanden wir empirische Evidenz für diese Idee (Schindler et al. 2019). In einer Studie ging es z. B. um Fleischkonsum. Hier machten die Versuchspersonen zunächst eine 10-minütige Achtsamkeitsübung oder ließen, in der Kontrollgruppe, ihre Gedanken frei schweifen. Um Schuldgefühle zu erzeugen, wurde den Versuchspersonen anschließend ein Video gezeigt, das die negativen Konsequenzen von Fleischkonsum für Tier und Umwelt dokumentiert. Danach wurde die Absicht erfasst, den Fleischkonsum in Zukunft zu reduzieren. Gemäß unserer Erwartung waren die Versuchspersonen in der Achtsamkeitsbedingung im Durchschnitt weniger bereit, ihren Fleischkonsum zu reduzieren, als die Versuchspersonen in der Kontrollgruppe. Neben diesen beiden Studien zeigten drei weitere Studien keine signifikanten Effekte, - wohlgemerkt, auch keinen positiven Effekt, wie es die Ergebnisse von Donald et al. (2019) nahelegen würden. Eine Meta-Analyse über alle fünf Studien hinweg stützt wiederum die Idee, dass Achtsamkeit Schuld-basierte moralische Reaktionen schwächt. Natürlich unterliegt auch die Interpretation dieser Ergebnisse gewissen Limitationen: Beispielsweise wurde kein tatsächliches Verhalten erfasst, es wurden nur kurze Achtsamkeitsübungen verwendet, und die Versuchspersonen hatten wenig Erfahrung mit solchen Übungen. Es ist Aufgabe zukünftiger Forschung, diese Punkte zu adressieren und die Validität dieser Idee weiter zu überprüfen.

Unter der empirisch gestützten Annahme, dass Achtsamkeit die Emotionsregulation fördert, kann man Achtsamkeit generell als Instrument für eine effiziente Zielerreichung sehen; vor allem, weil die Praxis - anders als im Buddhismus weitgehend säkularisiert und von ethischen Werten losgelöst ist. Man kann hier auch von nackter Achtsamkeit sprechen (Schindler et al. 2019). Dass eine wertfreie Achtsamkeitspraxis automatisch die Ziele und Werte einer Person einheitlich beeinflusst, ist theoretisch wenig plausibel und empirisch eine offene Frage. Bei Achtsamkeitsübungen in der Arbeitswelt oder im Militär geht es in erster Linie um Entspannung, Stressabbau und erhöhte Konzentration und weniger um das Kultivieren von Nächstenliebe und einem harmonischen Miteinander. 
Anzunehmen, dass Achtsamkeit ein Selbstläufer zu einer besseren und friedlicheren Welt sei, erscheint aus dieser Perspektive naiv. So zeigte eine gerade publizierte Studie, dass eine Achtsamkeitsintervention zwar zu mehr Zufriedenheit führt, aber nicht zu mehr umweltfreundlichem Konsum (Geiger et al. 2019). Das ist wenig überraschend, erscheint es theoretisch doch wesentlich plausibler, dass Achtsamkeit nur dann für die Umwelt nützlich ist, wenn Umweltschutz für die Person erstrebenswert ist und umweltschädliche Impulse besser reguliert werden können (z.B. Frustkonsum). Wenn Menschen aber gerne in den Urlaub fliegen, Rindfleisch essen oder einen neuen SUV kaufen möchten, dann würde die Idee von Schindler et al. (2019) nahelegen, dass man ein damit einhergehendes schlechtes Gewissen mit Achtsamkeitsübungen gut in den Griff bekommen kann. Das gilt ebenso bei einem Topmanager, der eine Massenentlassung von Angestellten umsetzen muss.

\section{Fazit}

Ein achtsamer Blick auf den aktuellen Forschungsstand zu Achtsamkeit führt zu dem Schluss, dass die Anzahl veröffentlichter Forschungsarbeiten zum Thema zwar beeindruckend hoch ist, valide Aussagen über die tatsächlichen Effekte von Achtsamkeit aber nach wie vor nur begrenzt möglich sind. Insbesondere wird die Einschätzung der empirischen Befundlage durch die Vielfalt an Konzeptualisierungen und Operationalisierungen erschwert. Verschiedene motivationale Anreize stehen dem Erkenntnisgewinn zusätzlich im Wege. Viele der genannten Limitationen sind jedoch für jedes Forschungsfeld eine Herausforderung und nicht achtsamkeitsspezifisch. Es ist auch klar, dass angewandte Forschung (wie im klinischen Bereich) und Grundlagenforschung sich in ihren Ansprüchen unterscheiden und niemals allen in gleichem Maße gerecht werden können. Allerdings ist es angesichts des aktuellen Achtsamkeits-Hypes umso wichtiger, deutlich auf vorhandene Schwachstellen hinzuweisen und höchste methodische Maßstäbe anzulegen. Hier ist in vielen Bereichen der Achtsamkeitsforschung Nachholbedarf.

Was für die empirische Ebene gilt, gilt auch für die theoretische. Die Vieldeutigkeit macht eine stringente Theoriebildung und -entwicklung kaum möglich. So wird Achtsamkeit in der Regel ohne größeren theoretischen Rahmen bzw. ohne eindeutige theoretische Herleitung untersucht. Das wird insbesondere in der Forschung zu prosozialen und unerwünschten Auswirkungen deutlich. Es ist theoretisch schwierig, eindeutig herzuleiten, warum Achtsamkeit generell, d. h. ohne Randbedingungen, automatisch Hilfeverhalten erhöht. Gleiches gilt für moralisches Verhalten. Wir sollten uns daher von dem Glauben verabschieden, dass Achtsamkeit die Welt automatisch für alle friedlicher und besser macht und ethische Werte und soziale Normen damit nicht länger diskutiert werden müssen. Es kann sogar die Frage gestellt werden, ob der Hype um Achtsamkeit nicht auch wegen stetig steigender Anforderungen einer (digitalen) Leistungsgesellschaft existiert, die immer mehr Belastbarkeit fordert. Diesem Umstand mit Achtsamkeitsübungen zu begegnen, hieße, sich an ein krankmachendes System anzupassen, anstatt das System selbst in Frage zu stellen und zu verändern. Passend dazu hat der Soziologe Hartmut Rosa die Achtsamkeitsbewegung als einen individualistischen Wellness-Trend beschrieben und ihr eine 
Subjektzentrierung und eine Entpolitisierung vorgeworfen (Stratmann 2016). Solche Ideen gehören dringend auf die (sozialpsychologische) Forschungsagenda.

All das bedeutet im Umkehrschluss nicht, dass Achtsamkeitsübungen keine Anwendung finden sollten. Als Instrument für effektive Emotionsregulation und Stressbewältigung und damit für eine Steigerung der Entspannung und der individuellen Lebenszufriedenheit scheint Achtsamkeit nach aktueller Studienlage einen substanziellen Beitrag leisten zu können. Auch für den zwischenmenschlichen Bereich kann Achtsamkeit einen Nutzen haben. Nur sollte die empirische Befundlage inklusive Randbedingungen und unerwünschter Nebenwirkungen genau geprüft werden, bevor Achtsamkeitsübungen in verschiedensten gesellschaftlichen Bereichen strukturell eingesetzt und in etlichen Ratgebern als ausnahmslos positiv beworben werden. Es scheint trivial, zu sagen, dass Achtsamkeit nicht die Antwort auf alle Probleme sein kann; angesichts des Hypes ist es das dann aber doch nicht.

Funding Open Access funding provided by Projekt DEAL.

Open Access Dieser Artikel wird unter der Creative Commons Namensnennung 4.0 International Lizenz veröffentlicht, welche die Nutzung, Vervielfältigung, Bearbeitung, Verbreitung und Wiedergabe in jeglichem Medium und Format erlaubt, sofern Sie den/die ursprünglichen Autor(en) und die Quelle ordnungsgemäß nennen, einen Link zur Creative Commons Lizenz beifügen und angeben, ob Änderungen vorgenommen wurden.

Die in diesem Artikel enthaltenen Bilder und sonstiges Drittmaterial unterliegen ebenfalls der genannten Creative Commons Lizenz, sofern sich aus der Abbildungslegende nichts anderes ergibt. Sofern das betreffende Material nicht unter der genannten Creative Commons Lizenz steht und die betreffende Handlung nicht nach gesetzlichen Vorschriften erlaubt ist, ist für die oben aufgeführten Weiterverwendungen des Materials die Einwilligung des jeweiligen Rechteinhabers einzuholen.

Weitere Details zur Lizenz entnehmen Sie bitte der Lizenzinformation auf http://creativecommons.org/ licenses/by/4.0/deed.de.

\section{Literatur}

Baer, R. A., Smith, G. T., Hopkins, J., Krietemeyer, J., \& Toney, L. (2006). Using self-report assessment methods to explore facets of mindfulness. Assessment, 13, 27-45.

Bergomi, C., Tschacher, W., \& Kupper, Z. (2013). The assessment of mindfulness with self-report measures: Existing scales and open issues. Mindfulness, 4, 191-202.

Bishop, S. R., Lau, M. A., Segal, Z. V., Buis, T., Anderson, N. D., Carlson, L., \& Devins, G. (2004). Mindfulness: a proposed operational definition. Clinical Psychology: Science and Practice, 11, 230-241.

Bosch, C., \& Michel, A. (2016). Achtsamkeit und Coaching. In S. Greif, H. Möller \& W. Scholl (Hrsg.), Handbuch Schlüsselkonzepte im Coaching. Berlin, Heidelberg: Springer.

Brown, K. W., \& Ryan, R. M. (2003). The benefits of being present: mindfulness and its role in psychological wellbeing. Journal of Personality and Social Psychology, 84, 822-848.

Brown, K.W., Creswell, J.D., \& Ryan, R.M. (2015). Handbook of mindfulness: theory, research, and practice. New York: Guilford.

Carter, E. C., Schönbrodt, F. D., Gervais, W. M., \& Hilgard, J. (2019). Correcting for bias in psychology: a comparison of meta-analytic methods. Advances in Methods and Practices in Psychological Science, $2,115-144$

Collard, P. (2016). Das kleine Buch vom achtsamen Leben: 10min am Tag für weniger Stress und mehr Gelassenheit. München: Heyne.

Condon, P., Desbordes, G., Miller, W. B., \& DeSteno, D. (2013). Meditation increases compassionate responses to suffering. Psychological Science, 24, 2125-2127. 
Coronado-Montoya, S., Levis, A. W., Kwakkenbos, L., Steele, R. J., Turner, E. H., \& Thombs, B. D. (2016). Reporting of positive results in randomized controlled trials of mindfulness-based mental health interventions. PLOS ONE, 11, e153220.

Creswell, J. D., Pacilio, L. E., Lindsay, E. K., \& Brown, K. W. (2014). Brief mindfulness meditation training alters psychological and neuroendocrine responses to social evaluative stress. Psychoneuroendocrinology, 44, 1-12.

Creswell, J.D. (2017). Mindfulness interventions. Annual Review of Psychology, 68, 491-516.

Van Dam, N. T., van Vugt, M. K., Vago, D. R., Schmalzl, L., Saron, C.D., Olendzki, A., Fox, K.C., et al. (2018). Mind the hype: a critical evaluation and prescriptive agenda for research on mindfulness and meditation. Perspectives on Psychological Science, 13, 36-61.

Dimidjian, S., \& Segal, S. V. (2015). Prospects for a clinical science of mindfulness-based intervention. American Psychologist, 70, 593-620.

Donald, J. N., Sahdra, B. K., Van Zanden, B., Duineveld, J. J., Atkins, P. W., Marshall, S. L., \& Ciarrochi, J. (2019). Does your mindfulness benefit others? A systematic review and meta-analysis of the link between mindfulness and prosocial behaviour. British Journal of Psychology, 110, 101-125.

Friese, M., \& Hofmann, W. (2016). State mindfulness, self-regulation, and emotional experience in everyday life. Motivation Science, 2, 1-14.

Geiger, S.M., Fischer, D., Schrader, U., \& Grossman, P. (2019). Meditating for the planet. Effects of a mindfulness-based intervention study on sustainable consumption behaviors. Environment \& Behavior. Advance online publication.

Gotink, R. A., Chu, P., Busschbach, J. J., Benson, H., Fricchione, G. L., \& Hunink, M. M. (2015). Standardised mindfulness-based interventions in healthcare: an overview of systematic reviews and metaanalyses of RCTs. PLOS ONE, 10, e0124344.

Graton, A., \& Ric, F. (2017). How guilt leads to reparation? Exploring the processes underlying the effects of guilt. Motivation and Emotion, 41, 343-352.

Grossman, P. (2019). On the porosity of subject and object in "mindfulness" scientific study: challenges to "scientific" construction, operationalization and measurement of mindfulness. Current Opinion in Psychology, 28, 102-107.

Hafenbrack, A. C., \& Vohs, K. D. (2018). Mindfulness meditation impairs task motivation but not performance. Organizational Behavior and Human Decision Processes, 147, 1-15.

Hülsheger, U.R., Alberts, H.J.E. M., Feinholdt, A., \& Lang, J.W. B. (2013). Benefits of mindfulness at work: the role of mindfulness in emotion regulation, emotional exhaustion, and job satisfaction. Journal of Applied Psychology, 98, 310-325.

Hülsheger, U.R., Feinholdt, A., \& Nübold, A. (2015). A low-dose mindfulness intervention and recovery from work: effects on psychological detachment, sleep quality, and sleep duration. Journal of Occupational and Organizational Psychology, 88, 464-489.

Jain, S., Shapiro, S. L., Swanick, S., Roesch, S. C., Mills, P. J., Bell, I., \& Schwartz, G. E. (2007). A randomized controlled trial of mindfulness meditation versus relaxation training: effects on distress, positive states of mind, rumination, and distraction. Annals of Behavioral Medicine, 33, 11-21.

Johnson, S. B., Park, H. S., Gross, C. P., \& James, B. Y. (2018). Complementary medicine, refusal of conventional cancer therapy, and survival among patients with curable cancers. JAMA Oncology, 4, 1375-1381.

Kabat-Zinn, J. (1982). An outpatient program in behavioral medicine for chronic pain patients based on the practice of mindfulness meditation: theoretical considerations and preliminary results. General Hospital Psychiatry, 4, 33-47.

Kabat-Zinn, J. (1990). Full catastrophe living: Using the wisdom of your body and mind to face stress, pain and illness. New York: Delacourt.

Kreplin, U., Farias, M., \& Brazil, I. A. (2018). The limited prosocial effects of meditation: a systematic review and meta-analysis. Scientific Reports, 8, 2403.

Lim, D., Condon, P., \& DeSteno, D. (2015). Mindfulness and compassion: an examination of mechanism and scalability. PLOS ONE, 10, e118221.

Long, E. C., \& Christian, M. S. (2015). Mindfulness buffers retaliatory responses to injustice: A regulatory approach. Journal of Applied Psychology, 100, 1409-1422.

Nilsson, H., \& Kazemi, A. (2016). Reconciling and thematizing definitions of mindfulness: the big five of mindfulness. Review of General Psychology, 20, 183-193.

Papies, E. K., Pronk, T.M., Keesman, M., \& Barsalou, L. W. (2015). The benefits of simply observing: mindful attention modulates the link between motivation and behavior. Journal of Personality and Social Psychology, 108, 148-170. 
Pfattheicher, S., Geiger, M., Hartung, J., Weiß, S., \& Schindler, S. (2017). Old wine in new bottles? The case of self-compassion and neuroticism. European Journal of Personality, 31, 160-169.

Pfattheicher, S., Sassenrath, C., \& Keller, J. (2019). Compassion magnifies third-party punishment. Journal of Personality and Social Psychology, 117, 124-141.

Popper, K. (1963). Conjectures and refutations. The growth of scientific knowledge. London: Routledge \& Kegan Paul.

Purser, R. (2019). Mcmindfulness: how mindfulness became the new capitalist spirituality. New York: Random House.

Pyszczynski, T., Solomon, S., \& Greenberg, J. (2015). Thirty years of terror management theory. Advances in Experimental Social Psychology, 52, 1-70.

Rosenkranz, M. A., Dunne, J. D., \& Davidson, R. J. (2019). The next generation of mindfulness-based intervention research: what have we learned and where are we headed? Current opinion in psychology, 28, 179-183.

Rosenthal, R. (1979). The file drawer problem and tolerance for null results. Psychological Bulletin, 86, 638-641.

Rupprecht, S., Koole, W., Chaskalson, M., Tamdjidi, C., \& West, M. (2019). Running too far ahead? Towards a broader understanding of mindfulness in organisations. Current Opinion in Psychology, 28, 32-36.

Salzberg, S. (1995). Loving-kindness: the revolutionary art of happiness. Boston: Shambala Publications.

Schindler, S., Pfattheicher, S., \& Reinhard, M.-A. (2019). Potential negative consequences of mindfulness in the moral domain. European Journal of Social Psychology, 49, 1055-1069.

Simmons, J. P., Nelson, L. D., \& Simonsohn, U. (2011). False-positive psychology: Undisclosed flexibility in data collection and analysis allows presenting anything as significant. Psychological Science, 22, 1359-1366.

Stratmann, B. (2016). Achtsamkeit löst die Probleme nicht - Interview mit Hartmut Rosa. Netzwerk Ethik heute. https://ethik-heute.org/achtsamkeit-loest-unsere-probleme-nicht/. Zugegriffen: 7.11.2016.

Strauss, C., Cavanagh, K., Oliver, A., \& Pettman, D. (2014). Mindfulness-based interventions for people diagnosed with a current episode of an anxiety or depressive disorder. A meta-analysis of randomised controlled trials. PLOS ONE, 9, e96110.

Tang, Y. Y., Hölzel, B.K., \& Posner, M.I. (2015). The neuroscience of mindfulness meditation. Nature Reviews Neuroscience, 16, 213-225.

Walsh, R., \& Shapiro, S.L. (2006). The meeting of meditative disciplines and Western psychology: a mutually enriching dialogue. American Psychologist, 61, 227-239.

Wilson, B. M., Mickes, L., Stolarz-Fantino, S., Evrard, M., \& Fantino, E. (2015). Increased false-memory susceptibility after mindfulness meditation. Psychological Science, 26, 1567-1573.

Zeidan, F., Emerson, N. M., Farris, S.R., Ray, J. N., Jung, Y., McHaffie, J.G., \& Coghill, R.C. (2015). Mindfulness meditation-based pain relief employs different neural mechanisms than placebo and sham mindfulness meditation-induced analgesia. Journal of Neuroscience, 35, 15307-15325.

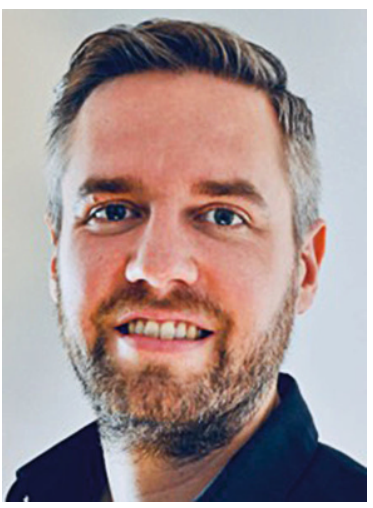

Dr. Simon Schindler Wissenschaftlicher Mitarbeiter an der Universität Kassel am Institut für Psychologie im Fachgebiet Sozialpsychologie; Tätigkeitsfelder: Forschung und Lehre, Forschungsschwerpunkte: Erkennen von Lüge und Wahrheit, Täuschungsverhalten, Bedrohungserleben, Achtsamkeit. 